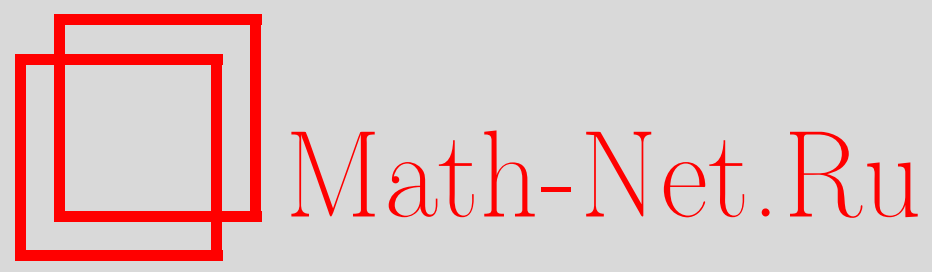

А. М. Седлецкий, О нулях преобразований Лапласа, Maтем. заметки, 2004, том 76, выпуск 6, 883-892

DOI: https://doi.org/10.4213/mzm160

Использование Общероссийского математического портала Math-Net.Ru подразумевает, что вы прочитали и согласны с пользовательским соглашением http://www . mathnet.ru/rus/agreement

Параметры загрузки:

IP: 18.208 .226 .222

26 апреля 2023 г., 14:40:05

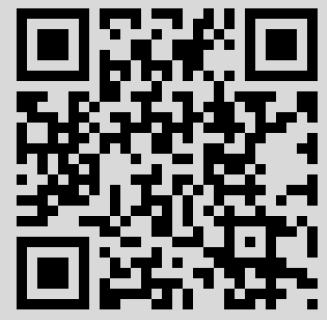


УДК 517.5

\section{О НУЛЯХ ПРЕОБРАЗОВАНИЙ ЛАПЛАСА}

\section{А. М. Седлецкий}

Пусть функция $f$ положительна, не убывает и интегрируема в интервале $(0,1)$. Тогда по теореме Пойа все нули преобразования Лапласа

$$
F(z)=\int_{0}^{1} e^{z t} f(t) d t
$$

лежат в левой полуплоскости $\operatorname{Re} z \leqslant 0$. В статье предполагается выполненньм дополнительное условие логарифмической вьпуклости $f$ в левой окрестности точки 1 . Найден вид (левой) криволинейной полуплоскости, а при условии $f(+0)>0$ - вид криволинейной полосы, содержащей все нули $F(z)$.

Библиография: 12 названий.

1. Введение. Рассматриваем преобразования Лапласа интегрируемых функций, сосредоточенных в конечном интервале, в качестве которого без ограничения общности берем интервал $(0,1)$. Итак, пусть

$$
F(z)=\int_{0}^{1} e^{z t} f(t) d t, \quad f \in L^{1}(0,1) .
$$

Такие (очевидно, целые) функции часто встречаются в различных разделах анализа, например, в спектральной теории, в теории дифференциально-разностных уравнений, при исследовании негармонических рядов Фурье и т.д. Большой интерес вызьвает вопрос о распределении нулей функций (1); он изучался в работах Харди [1], Пойа [2], Титчмарша [3], Картрайт [4], [5] и др. Для нас отправной точкой послужила следующая

Теорема А [2]. Пусть функиия $f$ интегрируема, положительна и не убывает в интервале $(0,1)$. Тогда все нули функиии $F(z)$ лежсат в левой полуплоскости $\operatorname{Re} z \leqslant 0 ;$ если при этом $f$ не является ступенчатой функиией вида

$$
f(t)=c_{i}, \quad t_{i}<t<t_{i+1}, \quad i=0, \ldots, n ; \quad t_{0}=0, \quad t_{n+1}=1, \quad 0<c_{i}<c_{i+1},
$$

Работа выполнена при поддержке Российского фонда фундаментальных исследований, грант № 03-01-00698, и программы "Ведущие научные школы", грант № НШ-680.2003.1. 
әде числа $t_{i}$ рациональны, то все нули функции $F(z)$ лежат в полуплоскости $\operatorname{Re} z<0$.

В классе функций $f$, удовлетворяющих условиям теоремы А, мы выделяем достаточно широкий подкласс и для него доказьваем более подробные утверждения о распределении нулей функций (1), а именно, утверждения о принадлежности всех нулей функций (1) вполне определенным собственным подмножествам множества $\operatorname{Re} z<0$. Дополнительное условие, задающее упомянутьй подкласс, состоит в том, что функция $f$ логарифмически выпукла в некоторой левой окрестности точки 1. Для этого подкласса найден вид (левой) криволинейной полуплоскости, а при условии $f(+0)>0$ - вид криволинейной полосы, содержащей все нули функции (1). В обоих случаях описание множества, содержащего нули, в известном смысле является точным. Кроме того, в статье доказано, что если $f$ логарифмически вьпукла на всем интервале $(0,1)$, то независимо от монотонности $f$, все нули функции $F(z)$ лежат на объединении горизонтальных полос $(2 n-1) \pi<|\operatorname{Im} z|<2 \pi n, n \in \mathbb{N}$.

2. Леммы. Функцию $f$, положительную в интервале $(a, b)$, назьвают логарифмически выпуклой в этом интервале, если функция $\log f$ вьпукла в $(a, b)$. Для дважды дифференцируемой положительной функции ее логарифмическая выпуклость в $(a, b)$ равносильна условию $\left(f^{\prime}\right)^{2} \leqslant f f^{\prime \prime}$ всюду в $(a, b)[6]$. В общем случае критерий логариффической вьпуклости дает

Лемма 1. Пусть функиия $f$ положительна в интервале $(a, b)$. Тогда для ее логарифмической выпуклости в этом интервале необходимо и достаточно, чтобы функиия

$$
f_{x}(t):=e^{x t} f(t)
$$

была выпуклой в $(a, b)$ при всех $x \in \mathbb{R}$.

ДокАЗАТЕЛЬСТво. Как в необходимой, так и в достаточной частях леммы мы имеем дело с непрерьвными функциями $f, \log f$. Будем пользоваться тем, что для функции $\varphi \in C(a, b)$ вьпуклость $\varphi$ в $(a, b)$ равносильна условию

$$
\varphi\left(\frac{t_{1}+t_{2}}{2}\right) \leqslant \frac{\varphi\left(t_{1}\right)+\varphi\left(t_{2}\right)}{2}
$$

для всех точек $t_{1}, t_{2} \in(a, b)[6]$. Тогда логарифмическая вьпуклость функции $f$ в $(a, b)$ равносильна условию

$$
f\left(\frac{t_{1}+t_{2}}{2}\right) \leqslant \sqrt{f\left(t_{1}\right) f\left(t_{2}\right)}
$$

для всех $t_{1}, t_{2} \in(a, b)$.

Пусть функция $f$ логарифмически выпукла в $(a, b)$. Тогда верно (3). Умножая (3) почленно на $\exp \left(x\left(t_{1}+t_{2}\right) / 2\right)$, получаем

$$
f_{x}\left(\frac{t_{1}+t_{2}}{2}\right) \leqslant \sqrt{f_{x}\left(t_{1}\right) f_{x}\left(t_{2}\right)} .
$$

Теперь применение к правой части неравенства Коши дает для $\varphi=f_{x}$ свойство (2), означающее выпуклость функции $f_{x}$. 
Пусть функция $f_{x}$ вьпукла в $(a, b)$. Тогда для $\varphi=f_{x}$ верно неравенство $(2)$, которое записьвается в виде

$$
f\left(\frac{t_{1}+t_{2}}{2}\right) \leqslant \frac{1}{2}\left(e^{x\left(t_{1}-t_{2}\right) / 2} f\left(t_{1}\right)+e^{x\left(t_{2}-t_{1}\right) / 2} f\left(t_{2}\right)\right) .
$$

По условию при фиксированных $t_{1}, t_{2}$ это неравенство выполняется для всех $x \in \mathbb{R}, \mathrm{a}$ в частности, и для значения $x$, при котором достигается минимум правой части. Дифференцированием находим, что для этого значения $x$ слагаемые в правой части (4) совпадают. Но тогда их полусумма равна их среднему геометрическому, и мы получаем свойство (3), означающее логарифмическую вьпуклость $f$. Лемма доказана.

ЛЕмма 2 [7]. Если функиия $\varphi$ интегрируема и выпукла в интервале $(0,2 \pi)$ (соответственно в интервале $(\pi / 2,5 \pi / 2))$, то

$$
\int_{0}^{2 \pi} \varphi(t) \cos t d t \geqslant 0 \quad\left(\text { соответственно } \quad \int_{\pi / 2}^{5 \pi / 2} \varphi(t) \sin t d t \geqslant 0\right) .
$$

Всюду в дальнейшем $x=\operatorname{Re} z, y=\operatorname{Im} z, r=|z|, \theta=\arg z$. Обозначим

$$
\Phi_{u}(z)=\int_{u}^{1} e^{-z t} g(t) d t .
$$

ЛЕмма 3. Пусть функиия $g$ имеет ограниченную вариацию на $\left[a_{0}, 1\right], 0 \leqslant a_{0}<1$, причем $g(1-0) \neq 0$. Тогда при любом фиксированном $b_{0} \in\left(a_{0}, 1\right)$ для $u \in\left[a_{0}, b_{0}\right]$ верна оченка

$$
\left|\Phi_{u}(z)\right| \geqslant C \frac{e^{-x}}{r}(1+o(1)), \quad x \rightarrow-\infty,
$$

әде $C>0$ от $u \in\left[a_{0}, b_{0}\right]$ не зависит.

ДокАЗАТЕЛЬСТво. Считаем, что $g(1)=g(1-0)$; по условию $g(1) \neq 0$. Пользуясь непрерьвностью $g$ в точке 1 слева, фиксируем $\delta \in\left(0,1-b_{0}\right)$ столь малым, чтобы

$$
\operatorname{var}(g(t): 1-\delta \leqslant t \leqslant 1) \leqslant \frac{|g(1)|}{2} .
$$

Интегрируем по частям

$$
\Phi_{u}(z)=-\frac{1}{z}\left(e^{-z} g(1)-e^{-z u} g(u)-\int_{u}^{1} e^{-z t} d g(t)\right) .
$$

Последний интеграл разобьем на слагаемые $K_{1}$ и $K_{2}$, отвечающие соответственно отрезкам $[u, 1-\delta]$ и $[1-\delta, 1]$. При $x \leqslant 0$ с учетом $(7)$ для них получаем оценки

$$
\left|K_{1}\right| \leqslant V e^{-(1-\delta) x}, \quad\left|K_{2}\right| \leqslant \frac{|g(1)|}{2} e^{-x},
$$

которые вместе с неравенством треугольника, примененным к (8), дают неравенство

$$
\left|\Phi_{u}(z)\right| \geqslant \frac{|g(1)|}{2} \frac{e^{-x}}{r}\left(1-M e^{\left(1-b_{0}\right) x}-V e^{\delta x}\right), \quad x \leqslant 0,
$$

означающее справедливость леммы 3 . 


\section{3. Правая граница для нулей.}

Теорема 1. Пусть выполнены условия теоремы А, и пусть, кроме того, функция $f$ логарифмически выпукла на некотором интервале $(1-a, 1), 0<a \leqslant 1$. Тогда все нули функиии (1) с достаточно большим модулем мнимой части лежат на множестве

$$
x \leqslant-\log \left(y^{2} \cdot \int_{0}^{\pi /(2|y|)} t f(1-t) d t\right)+C, \quad|y|>y_{0},
$$

где $C$ - некоторая постоянная.

Заметим, что содержательность теоремы 1 (в сравнении с теоремой А) проявляется в случае $f(1-0)=+\infty$, когда

$$
y^{2} \int_{0}^{\pi /(2 y)} t f(1-t) d t>\frac{\pi^{2}}{8} f\left(1-\frac{\pi}{2 y}\right) \rightarrow+\infty, \quad y \rightarrow+\infty .
$$

ДоКАЗАТЕЛЬСТво ТЕОРЕМЫ 1 . Так как $F(\bar{z})=\bar{F}(z)$, то достаточно рассмотреть $y>0$. Имеем

$$
\begin{gathered}
e^{-z} F(z)=\int_{0}^{1} e^{-z t} f(1-t) d t=: G(z), \\
V(z):=-\operatorname{Im} G(z)=\int_{0}^{1} e^{-x t} f(1-t) \sin y t d t .
\end{gathered}
$$

Оценим снизу $|V(z)|$ при достаточно больших $y$ и при $x<0$ (напомним, что по теореме $\mathrm{A}$ при $x \geqslant 0$ нулей у $F(z)$ нет). Обозначим

$$
N=N(y):=\max \left(n \in \mathbb{N}: \frac{\pi}{2 y}+\frac{2 \pi n}{y} \leqslant a\right), \quad a_{N}:=\frac{\pi}{2 y}+\frac{2 \pi N}{y}
$$

и заметим, что $a_{N} \leqslant a$. Кроме того, так как $\pi /(2 y)+2 \pi(N+1) / y>a$, то $a_{N}>a-2 \pi / y$, и потому

$$
\frac{a}{2}<a_{N} \leqslant a
$$

при достаточно больших $y$. Пусть

$$
V(z)=\left(\int_{0}^{\pi /(2 y)}+\int_{\pi /(2 y)}^{a_{N}}+\int_{a_{N}}^{1}\right) e^{-x t} f(1-t) \sin y t d t=: V_{1}+V_{2}+V_{3} .
$$

Так как $x<0$, то

$$
V_{1}>\frac{2}{\pi} y \int_{0}^{\pi /(2 y)} t f(1-t) d t
$$

Далее, по условию функция $f(1-t)$ логарифмически вьпукла на $(0, a)$. По лемме 1 функция $e^{-x t} f(1-t)$ вьпукла на $(0, a)$, и значит, по лемме $2 V_{2} \geqslant 0$. А так как

$$
\begin{aligned}
V_{3} & =-\operatorname{Im} \int_{a_{N}}^{1} e^{-z t} f(1-t) d t=\operatorname{Im}\left(\frac{1}{z} \int_{a_{N}}^{1} f(1-t) d e^{-z t}\right) \\
& =\operatorname{Im}\left(\frac{1}{z}\left(f(+0)-f\left(1-a_{N}\right) e^{-z a_{N}}-\int_{a_{N}}^{1} e^{-z t} d f(1-t)\right)\right),
\end{aligned}
$$


то с учетом (11) при $x<0$ имеем

$$
\left|V_{3}\right| \leqslant A \frac{e^{-x}}{r} \leqslant A \frac{e^{-x}}{y}, \quad A=3 f\left(1-\frac{a}{2}\right)
$$

У функции $G(z)$, а значит, и у функции $F(z)$, нет нулей там, где $V_{1}+V_{2}>\left|V_{3}\right|$. Поэтому из полученных оценок для $V_{i}, i=1,2,3$, следует, что у $F(z)$ нет нулей там, где

$$
\frac{2}{\pi} y \int_{0}^{\pi /(2 y)} t f(1-t) d t>\frac{A}{y} e^{-x}, \quad y>y_{0}>0 .
$$

Логарифмируя, получаем утверждение теоремы 1.

Из теоремы 1 и из содержащегося в (9) неравенства с учетом теоремы А вытекает

СлЕДСТВИЕ 1. Пусть выполнены условия теоремы 1. Тогда все нули функиии $F(z)$ лежст на обвединении множества

$$
x \leqslant \min \left(0,-\log f\left(1-\frac{\pi}{2|y|}\right)+C\right), \quad|y|>\frac{\pi}{2},
$$

әде $C$ - некоторая постоянная, с полуполосой $x<0,|y| \leqslant \pi / 2$.

Обозначим через

$$
\left(z_{n}\right)_{1}^{\infty} \quad\left(\text { где } \forall n \quad\left|z_{n+1}\right| \geqslant\left|z_{n}\right|\right)
$$

последовательность всех нулей функции $F(z)$ (хорошо известно, см., например, [8], что функция (1) имеет бесконечное множество нулей). Заинтересуемся условиями на $f$, при которых

$$
\operatorname{Re} z_{n} \rightarrow-\infty, \quad n \rightarrow \infty
$$

В статье [9] доказано, что в условиях теоремы А все нули функции $F(z)$ лежат в некоторой правой полуплоскости $\operatorname{Re} z \geqslant h>-\infty$ (т.е. в вертикальной полосе $h \leqslant \operatorname{Re} z \leqslant 0$ ) тогда и только тогда, когда $0<f(+0) \leqslant f(1-0)<+\infty$. Таким образом, для справедливости соотношения (12) необходимо выполнение хотя бы одного из условий $f(+0)=0$, $f(1-0)=+\infty$. Из следствия 1 вытекает

СлЕДСтвИЕ 2. Пусть функиия $f$ удовлетворяет условиям теоремы 1. Тогда если $f(1-0)=+\infty$, то для нулей функиии $F(z)$ выполняется соотношение (12).

4. Левая граница для нулей. Она зависит от поведения функции $f$ в окрестности точки 0. Мы примем условие $f(+0)>0$.

ТЕОрема 2. Пусть выполнены условия теоремы 1 и, кроме того, $f(+0)>0$. Тогда найдутся постоянные $C \in \mathbb{R}, H, y_{0}>0$ такие, что все нули функиии $F(z)$ лежат на обвединении множества

$$
x \geqslant-\log \left(|y| \int_{0}^{\pi /(2|y|)} f(1-t) d t\right)+C, \quad|y|>y_{0}
$$

смиожеством $x \geqslant-H,|y| \leqslant y_{0}$. 
ДокАЗАТЕЛЬСтво. Пусть $G(z)$ и $a_{N}-$ те же, что в доказательстве теоремы 1 . Достаточно рассматривать $y \geqslant 0$. Сначала покажем, что в любом секторе $\cos \theta \leqslant-\delta<0$ содержится не более конечного числа нулей функции $G(z)$. Для этого обозначим через $J_{1}, J_{2}$ слагаемые в интеграле $(10)$, отвечающие интервалам $(0,1 / 2)$ и $(1 / 2,1)$ соответственно. Тогда

$$
\left|J_{1}\right| \leqslant e^{-x / 2}\|f\|_{1}, \quad x \leqslant 0,
$$

а так как $J_{2}=\Phi_{1 / 2}$ в обозначении (5), то для $J_{2}$ по лемме $3($ с $g(t)=f(1-t))$ верна оценка (6). Значит, если $H$ достаточно велико, то по неравенству треугольника для $x \leqslant-H<0$ получаем оценку

$$
|G(z)| \geqslant C_{1} \frac{e^{-x}}{r}-C_{2} e^{-x / 2} \geqslant \frac{C_{1}}{r} e^{-x}\left(1-C_{3} r e^{x / 2}\right) .
$$

Последняя скобка положительна, если $\cos \theta \leqslant-\delta<0$, a $r$ достаточно велико, и промежуточное утверждение, касающееся сектора, доказано.

Таким образом, для отыскания левой границы для нулей можно рассматривать только множество

$$
x<-H, \quad y>\frac{r}{2},
$$

где $H$ достаточно велико. Можно считать, что $a<1$. Положим

$$
G(z)=\left(\int_{0}^{a_{N}}+\int_{a_{N}}^{1}\right) e^{-z t} f(1-t) d t=: G_{1}(z)+G_{2}(z) .
$$

С учетом (11) по лемме 3 имеем оценку (6) для $\Phi_{a_{N}}(z)=G_{2}(z)$. Значит, на множестве (13) при достаточно большом $H$ верна оценка

$$
\left|G_{2}(z)\right| \geqslant B \frac{e^{-x}}{y}, \quad B>0
$$

Пусть теперь $G_{1}=U-i V$, где

$$
U=\int_{0}^{a_{N}} e^{-x t} f(1-t) \cos y t d t, \quad V=\int_{0}^{a_{N}} e^{-x t} f(1-t) \sin y t d t .
$$

Запишем

$$
V=\int_{0}^{\pi / y}+\int_{\pi / y}^{3 \pi /(2 y)}+\int_{3 \pi /(2 y)}^{a_{N}-\pi / y}+\int_{a_{N}-\pi / y}^{a_{N}}=: V_{1}+V_{2}+V_{3}+V_{4} .
$$

Тогда $V_{2}<0$ в силу отрицательности $\sin y t$, а последовательное применение лемм 1,2 дает неравенство $V_{3} \leqslant 0$. Благодаря невозрастанию функции $f(1-t)$ и тому, что $-x / y \leqslant M_{1}<+\infty$ на множестве (13), имеем

$$
0<V_{1}<2 e^{-\pi x / y} \int_{0}^{\pi /(2 y)} f(1-t) d t<C \int_{0}^{\pi /(2 y)} f(1-t) d t .
$$


Далее, учитьвая (11) и монотонность $f$, а также обозначая $I_{N}=\left(a_{N}-\pi / y, a_{N}\right)$ и $C_{1}=3 f(1-a / 2)$, при $y>y_{0}$ получаем

$$
\begin{aligned}
\left|V_{4}\right| & =\left|\operatorname{Im}\left(\frac{1}{z} \int_{I_{N}} f(1-t) d e^{-z t}\right)\right| \\
& \leqslant \frac{1}{r}\left|f\left(1-a_{N}\right) e^{-z a_{N}}-f\left(1-a_{N}+\frac{\pi}{y}\right) e^{-z\left(a_{N}-\pi / y\right)}-\int_{I_{N}} e^{-z t} d f(1-t)\right| \\
& <C_{1} \frac{e^{-a x}}{y}
\end{aligned}
$$

Ho

$$
V=\int_{0}^{\pi /(2 y)}+\int_{\pi /(2 y)}^{a_{N}}>0
$$

так как в первом слагаемом подынтегральная функция положительна, а ко второму, благодаря лемме 1 , применима лемма 2 . И так как $V_{2}, V_{3} \leqslant 0$, то

$$
0<V=\left(V_{1}+V_{4}\right)+\left(V_{2}+V_{3}\right) \leqslant V_{1}+V_{4} \leqslant V_{1}+\left|V_{4}\right|
$$

т.e.

$$
|V| \leqslant C \int_{0}^{\pi /(2 y)} f(1-t) d t+C_{1} \frac{e^{-a x}}{y} .
$$

Аналогично поступаем с функцией $U$. Записываем

$$
U=\int_{0}^{\pi /(2 y)}+\int_{\pi /(2 y)}^{\pi / y}+\int_{\pi / y}^{a_{N}-3 \pi /(2 y)}+\int_{a_{N}-3 \pi /(2 y)}^{a_{N}}=: U_{1}+U_{2}+U_{3}+U_{4}
$$

и замечаем, что $U_{2}<0$ в силу отрищательности $\cos y t$, a $U_{3} \leqslant 0$ по леммам 1,2 . Далее, на множестве (13)

$$
0<U_{1}<e^{-\pi x /(2 y)} \int_{0}^{\pi /(2 y)} f(1-t) d t<C \int_{0}^{\pi /(2 y)} f(1-t) d t
$$

а для $\left|U_{4}\right|$ верна оценка типа (15), т.е. $\left|U_{4}\right| \leqslant C_{1} e^{-a x} / y$. Подобно (16), верно неравенство

$$
U=\int_{0}^{a_{N}-\pi /(2 y)}+\int_{a_{N}-\pi /(2 y)}^{a_{N}}>0
$$

и значит, для $|U|$ мы получаем оценку (17).

Теперь вспоминая, что $G=U-i V+G_{2}$, с помощью оценок (14) и (17) (последняя верна и для $|U|)$, находим

$$
|G(z)| \geqslant B \frac{e^{-x}}{y}-C_{2} \int_{0}^{\pi /(2 y)} f(1-t) d t-C_{3} \frac{e^{-a x}}{y} .
$$

Если $H$ достаточно велико, то $(B / 2) e^{-x}>C_{3} e^{-a x}$ при $x<-H$, и из (18) следует, что у $G(z)$, а значит, и у $F(z)$, нет нулей на множестве

$$
\frac{B}{2} e^{-x}>C_{2} y \int_{0}^{\pi /(2 y)} f(1-t) d t, \quad y>y_{0} .
$$

Теорема 2 доказана.

C учетом теоремы $\mathrm{A}$ теоремы 1,2 дают 
СлЕДСТВИЕ 3. Пусть выполнены условия теоремы 2. Тогда найдутся постоянные $C_{1}, C_{2} \in \mathbb{R}, H, y_{0}>0$ такие, что все нули функиии $F(z)$ лежсат в криволинейной полосе, являющейся обгединением мнохества

$$
-\log \left(|y| \int_{0}^{\pi /(2|y|)} f(1-t) d t\right)+C_{1} \leqslant x \leqslant-\log \left(y^{2} \int_{0}^{\pi /(2|y|)} t f(1-t) d t\right)+C_{2}<0
$$

где $|y|>y_{0}$, с множеством $-H \leqslant x<0,|y| \leqslant y_{0}$.

ЗАмЕчАнИЕ 1. Благодаря следствию 1 , множество (19) в следствии 3 может быть заменено множеством

$$
-\log \left(|y| \int_{0}^{\pi /(2|y|)} f(1-t) d t\right)+C_{1} \leqslant x \leqslant-\log f\left(1-\frac{\pi}{2|y|}\right)+C_{2}<0 .
$$

5. Случай правильного изменения функции $f$. Для функции $f$, правильно меняющейся в окрестности точки 1 , асимптотика входящих в (19) интегралов вычисляется, что дает возможность упростить вид множества (19).

Приводимые ниже определения и факты взяты из [10].

Функция $g$, положительная и измеримая в правой окрестности точки 0 , назьвается правильно меняющейся функиией (п.м.ф.) порядка $\alpha \in \mathbb{R}$, если для всех $\lambda>0$

$$
\lim _{t \rightarrow+0} \frac{g(\lambda t)}{g(t)}=\lambda^{\alpha}
$$

П.м.ф. порядка $\alpha=0$ назьвается медленно меняющейся функцией (м.м.ф.). П.м.ф. порядка $\alpha$ имеет вид $g(t)=t^{\alpha} l(t)$, где $l(t)$ - м.м.ф. Для любой м.м.ф. $l(t)$ найдется эквивалентная ей при $t \rightarrow+0$, непрерьвно дифференцируемая функция $l_{0}(t)$ с условием

$$
l_{0}^{\prime}(t)=o\left(\frac{l_{0}(t)}{t}\right), \quad t \rightarrow+0 .
$$

Обозначим

$$
g_{1}(\varepsilon)=\frac{1}{\varepsilon} \int_{0}^{\varepsilon} g(t) d t, \quad g_{2}(\varepsilon)=\frac{1}{\varepsilon^{2}} \int_{0}^{\varepsilon} t g(t) d t .
$$

Лемма 4. Пусть функиия $g$ интегрируема на $(0,1)$ и является п.м.ф. порядка $\alpha>-1$. Тогда при $\varepsilon \rightarrow+0$

$$
g_{1}(\varepsilon) \sim \frac{1}{1+\alpha} g(\varepsilon), \quad g_{2}(\varepsilon) \sim \frac{1}{2+\alpha} g(\varepsilon) .
$$

ДокАЗАТЕЛЬСТво. По условию $g(t)=t^{\alpha} l(t)$, где $l(t)$ - м.м.ф. Так как замена функции $g$ на ей эквивалентную при $t \rightarrow+0$ не повлияет на асимптотику функций $g_{1}, g_{2}$, то мы можем считать, что для $l(t)$ выполнено свойство (21). По правилу Лопиталя

$$
\begin{aligned}
\lim _{\varepsilon \rightarrow+0} \frac{g_{1}(\varepsilon)}{g(\varepsilon)} & =\lim _{\varepsilon \rightarrow+0} \frac{1}{\varepsilon^{1+\alpha} l(\varepsilon)} \int_{0}^{\varepsilon} t^{\alpha} l(t) d t \\
& =\lim _{\varepsilon \rightarrow+0} \frac{\varepsilon^{\alpha} l(\varepsilon)}{(1+\alpha) \varepsilon^{\alpha} l(\varepsilon)+\varepsilon^{1+\alpha} l^{\prime}(\varepsilon)}=\lim _{\varepsilon \rightarrow+0} \frac{1}{1+\alpha+\left(\varepsilon l^{\prime}(\varepsilon) / l(\varepsilon)\right)},
\end{aligned}
$$

откуда с учетом свойства (21) следует требуемая асимптотика для $g_{1}$. При замене $g_{1}$ на $g_{2}$ в $(22) \alpha$ заменяется на $\alpha+1$, и все повторяется. Лемма доказана.

Вернемся к следствию 3. Выражения под знаками логарифмов в (19) пропорциональны соответственно $g_{1}(\varepsilon)$ и $g_{2}(\varepsilon)$, где $\varepsilon=\pi /(2|y|)$, а $g(t)=f(1-t)$. Поэтому с помощью леммы 4 и соотношения (20) из следствия 3 вытекает 
СЛЕДСТВИЕ 4. Пусть выполнены условия теоремы 2, и пусть, кроме того, функиия $f(1-t)$ является правильно меняющейся функиией порядка $\alpha \in(-1,0)$. Тогда все нули функиии $F(z)$ лежат в криволинейной полосе, являющейся обтединением множества $-H \leqslant x<0,|y| \leqslant y_{0}$ с множеством

$$
\left|x+\log f\left(1-\frac{1}{|y|}\right)\right| \leqslant C, \quad|y|>y_{0}
$$

Пример функции $f(1-t)=1 /\left(t \log ^{2} t\right)$, когда $g_{1}(\varepsilon)=1 /(\varepsilon|\log \varepsilon|)$, показывает, что при $\alpha=-1$ функции $g_{1}$ и $g_{2}$ уже не являются асимптотически пропорциональными, и в этом случае мы вынуждены довольствоваться замечанием 1.

\section{6. Распределение нулей в горизонтальных полосах.}

ТЕОремА 3. Пусть функиия $f$ интегрируема и логарифмически выпукла в интервале $(0,1)$. Тогда все нули функиии (1) лехст на облединении горизонтальных полос

$$
(2 n-1) \pi<|\operatorname{Im} z|<2 \pi n, \quad n \in \mathbb{N} .
$$

ДОКАЗАТЕЛЬСТВО. Пусть

$$
G(z)=e^{-z} F(z)=\int_{0}^{1} e^{-z t} f(1-t) d t=: U(z)-i V(z)
$$

Достаточно показать, что у функции $G(z)$ нет нулей в полосах

$$
2 \pi n \leqslant y \leqslant(2 n+1) \pi, \quad n \in \mathbb{Z}_{+} .
$$

Пусть сначала

$$
\left(2 n+\frac{1}{2}\right) \pi \leqslant y \leqslant(2 n+1) \pi, \quad n \in \mathbb{Z}_{+} .
$$

Если $n=0$, то $\sin y t>0$ при $0<t<1$, и так как $f>0$, то $V>0$. Если $n \geqslant 1$, то, подразумевая $e^{-x t} f(1-t) \sin y t$ в качестве подьнтегральной функции, запишем

$$
V(z)=\int_{0}^{\pi /(2 y)}+\sum_{k=0}^{n-1} \int_{\pi(1 / 2+2 k) / y}^{\pi(5 / 2+2 k) / y}+\int_{\pi(1 / 2+2 n) / y}^{1}=: I_{1}+\sum_{k=1}^{n-1} V_{k}+I_{2} .
$$

Kaк в $I_{1}$, так и в $I_{2}$, имеем $\sin y t>0$; для $I_{1}$ это очевидно, а если рассматривается $I_{2}$, то $\pi(1 / 2+2 n) / y<t<1$, и значит, $\pi / 2+2 \pi n<y t<y \leqslant(2 n+1) \pi$ (мы учли правое неравенство (23)). Следовательно, $I_{1}+I_{2}>0$. А последовательное применение лемм 1 и 2 дает неравенство $V_{k} \geqslant 0$. Значит, $V>0$ на множестве $(23)$, и на этом множестве нет нулей $G(z)$.

Остается рассмотреть полосы

$$
2 \pi n \leqslant y<\frac{\pi}{2}+2 \pi n, \quad n \in \mathbb{Z}_{+\cdot}
$$


Здесь аналогичную работу продельваем уже с функцией $U(z)$. Если $n=0$, то $\cos y t>0$ для $0<t<1$, и поэтому $U>0$. При $n \geqslant 1$ запишем

$$
U(z)=\sum_{k=1}^{n} \int_{2 \pi(k-1) / y}^{2 \pi k / y}+\int_{2 \pi n / y}^{1}=: \sum_{k=1}^{n} U_{k}+J
$$

с подынтегральной функцией $e^{-x t} f(1-t) \cos y t$. Снова $U_{k} \geqslant 0$ по леммам 1 и 2, а $J>0$ из-за положительности подьнтегральной функции: действительно, если $2 \pi n / y<t<1$, то в силу правого неравенства (24) имеем $2 \pi n<y t<y \leqslant \pi / 2+2 \pi n$. Значит, $U>0$ на множестве (24), и на этом множестве у $G(z)$ нулей также нет. Теорема 3 доказана.

Из теорем А и 3 вытекает

СледСТВИЕ 5. Пусть функиия $f$ интегрируема, не убывает и логарифмически выпукла в интервале $(0,1)$. Тогда все нули функиии (1) лежат на обвединении полуполос

$$
\operatorname{Re} z<0, \quad(2 n-1) \pi<|\operatorname{Im} z|<2 \pi n, \quad n \in \mathbb{N} .
$$

В качестве примера рассмотрим функцию типа Mиттаг-Леффлера порядка единица

$$
E_{1}(z ; \mu)=\frac{1}{\Gamma(\mu-1)} \int_{0}^{1} e^{z t}(1-t)^{\mu-2} d t, \quad \mu>1 .
$$

(см. [11]). При $1<\mu<2$ для функции $f(t)=(1-t)^{\mu-2}$ выполняются условия теоремы 3 . Известно [12], что при $1<\mu<2$ все нули функции $E_{1}(z ; \mu)$ лежат в полуплоскости $\operatorname{Re} z<\mu-2$. Отсюда и из теоремы 3 вытекает

СлЕДСТВИЕ 6 . При $1<\mu<2$ все нули функиии $E_{1}(z ; \mu)$ лежат на обвединении полуполос

$$
\operatorname{Re} z<\mu-2, \quad(2 n-1) \pi<|\operatorname{Im} z|<2 \pi n, \quad n \in \mathbb{N} \text {. }
$$

\section{СПИСОК ЦИТИРОВАННОЙ ЛИТЕРАТУРЫ}

[1] Hardy G. H On the zeroes of certain classes of integral Taylor series II // Proc. London Math. Soc. Ser. 2. 1905. V. 2. P. 401-431.

[2] Polya G. Über die Nullstellen gewisser ganzer Funktionen // Math. Z. 1918. V. 2. P. 352-383.

[3] Titchmarsh E. C. The zeros of certain integral functions // Proc. London Math. Soc. Ser. 2. 1926. V. 25. P. 283-302.

[4] Cartwright M. The zeros of certain integral functions // Quart. J. Math. 1930. V. 1. P. 38-59.

[5] Cartwright M. The zeros of certain integral functions (II) // Quart. J. Math. 1931. V. 2. P. 113-129.

[6] Харди Г. Г., Литтлвуд Д. Е., Полиа Г. Неравенства. М.: ИЛ, 1948.

[7] Харди Г. Г., Рогозинский В. В. Ряды Фурье. М.: Физматгиз, 1959.

[8] Левин Б. Я. Целые функции (курс лекций). М.: МГУ, 1971.

[9] Sedletskii A. M. On zeros of Laplace transforms of finite measure // Integral Transforms and Special Functions. 1993. V. 1. P. 51-59.

[10] Сенета Е. Правильно меняющиеся функции. М.: Наука, 1985.

[11] Джрбашян М. М. Интегральные преобразования и представления функций в комплексной области. М.: Наука, 1966.

[12] Седлецкий А. М. О нулях функции типа Миттаг-Леффрера // Матем. заметки. 2000. Т. 68 . № 5. C. $710-724$. 